\title{
Cosmic Ray Energy Spectrum from Measurements of Air Showers
}

\author{
T. K. Gaisser ${ }^{1}$, T. Stanev ${ }^{1}$ \& S. Tilav ${ }^{1}$ \\ ${ }^{1}$ Bartol Research Institute and Department of Physics and Astronomy, \\ University of Delaware, Newark, DE 19716, USA.
}

\begin{abstract}
This review focuses on high-energy cosmic rays in the PeV energy range and above. Of particular interest is the knee of the spectrum around $3 \mathrm{PeV}$ and the transition from cosmic rays of Galactic origin to particles from extra-galactic sources. Our goal is to establish a baseline spectrum from $10^{14}$ to $10^{20} \mathrm{eV}$ by combining the results of many measurements at different energies. In combination with measurements of the nuclear composition of the primaries, the shape of the energy spectrum places constraints on the number and spectra of sources that may contribute to the observed spectrum.
\end{abstract}

PACS numbers: ...

\section{INTRODUCTION}

In the 100 years since Victor Hess's balloon flight that marks the discovery of cosmic rays, a great deal has been learned about their composition, propagation and sources. We know that most particles originate from sources in the local galaxy, having spent on average $10^{7}$ years in diffusive motion in the interstellar medium (ISM) and the galactic halo before being lost to intergalactic space 1]. We know that the power requirement to maintain the intensity of cosmic rays at a near constant value is a few per cent of the energy available in supernova explosions and that there is a well developed theory of non-linear, diffusive acceleration by shocks driven by expanding supernova remnants (SNR) 2]. We know that diffusion in the interstellar medium depends on energy in such a way that higher energy nuclei escape more quickly than lower energy ones, but the shape of this energy dependence is still uncertain at high energy [3].

Measurements of diffuse gamma radiation from $<1$ to $>100 \mathrm{GeV}$ by the Fermi Satellite [4] confirm this general picture of the origin and propagation of galactic cosmic rays. In the $\mathrm{TeV}$ range, ground based gamma-ray telescopes have observed emission of $>\mathrm{TeV}$ gamma-rays from supernova remnants, as expected if there is particle acceleration to energies of $>10 \mathrm{TeV}[\underline{5}$. However, because electrons are more efficient radiators than protons, the relation of these observations to the fluxes of high-energy protons and nuclei observed at Earth is not completely clear. In particular, the maximum energy to which protons and nuclei can be accelerated by supernova remnants is not yet directly established.

The picture outlined above is driven by direct measurements of cosmic rays with detectors near the top or outside of the atmosphere. Measurements with spectrometers in high-altitude balloons and satellites measure nuclear and isotopic composition in the GeV energy range and spectra of individual elements up to $\mathrm{TeV} /$ nucleon [6]. Instruments with calorimeters and transition radiation detectors extend the energy measurements to $\sim 100 \mathrm{TeV}$ per nucleus. Recent measurements by CREAM [7, 8] and PAMELA [9] support earlier observations by JACEE [10, 11] and ATIC [12] that the spec- trum of helium is somewhat harder than that of protons. The PAMELA measurements show that the spectrum of both protons and helium become harder above a rigidity of $200 \mathrm{GV}$. The CREAM data suggest that this is also the case for heavier nuclei [8]. Further new data are expected soon from the AMS-2 detector that by now has worked on the International Space Station for more than one year.

The cosmic-ray flux above $100 \mathrm{TeV}$ amounts to about 5 particles per square meter per steradian per day. Therefore, direct measurement of the cosmic-ray spectrum above this energy with balloon borne or satellite detectors is difficult. The highest direct measurement so far is still the series of measurements by Grigorov et al. carried on Proton satellites 13], which extends to $\sim \mathrm{PeV}$. Data for the spectrum above a few hundred $\mathrm{TeV}$ comes from large air shower arrays on the ground. The air-shower experiments observe the cascades of secondary particles in the atmosphere initiated by the interaction of the high energy primary particles. In such indirect measurements, the information about composition is limited (at best) to determining the relative abundances of the main groups of nuclei. Moreover, the relation between the observed signal at the ground and the primary energy depends on the mass of the primary nucleus. In addition, the relation between the observed signal and the primary energy depends on the model(s) of hadronic interactions used to interpret the cascades. Given the large uncertainties in energy assignment, it is not surprising that different measurements give different results in the same or overlapping energy regions. Another complexity is that techniques for measuring the shower at the ground differ from one experiment to the next (for example, scintillators compared to water Cherenkov tanks). In this review we therefore use observed features in the energy spectra to cross-calibrate results from different arrays.

Since air shower measurements are calorimetric in nature, the natural energy variable to use is total energy per nucleus. This contrasts with the direct measurements, which generally use energy per nucleon. The latter is the natural variable for studying propagation of nuclei because energy per nucleon is conserved in spallation. A variable different from both of these is relevant for interpreting observed spectra. This is magnetic rigidity, 


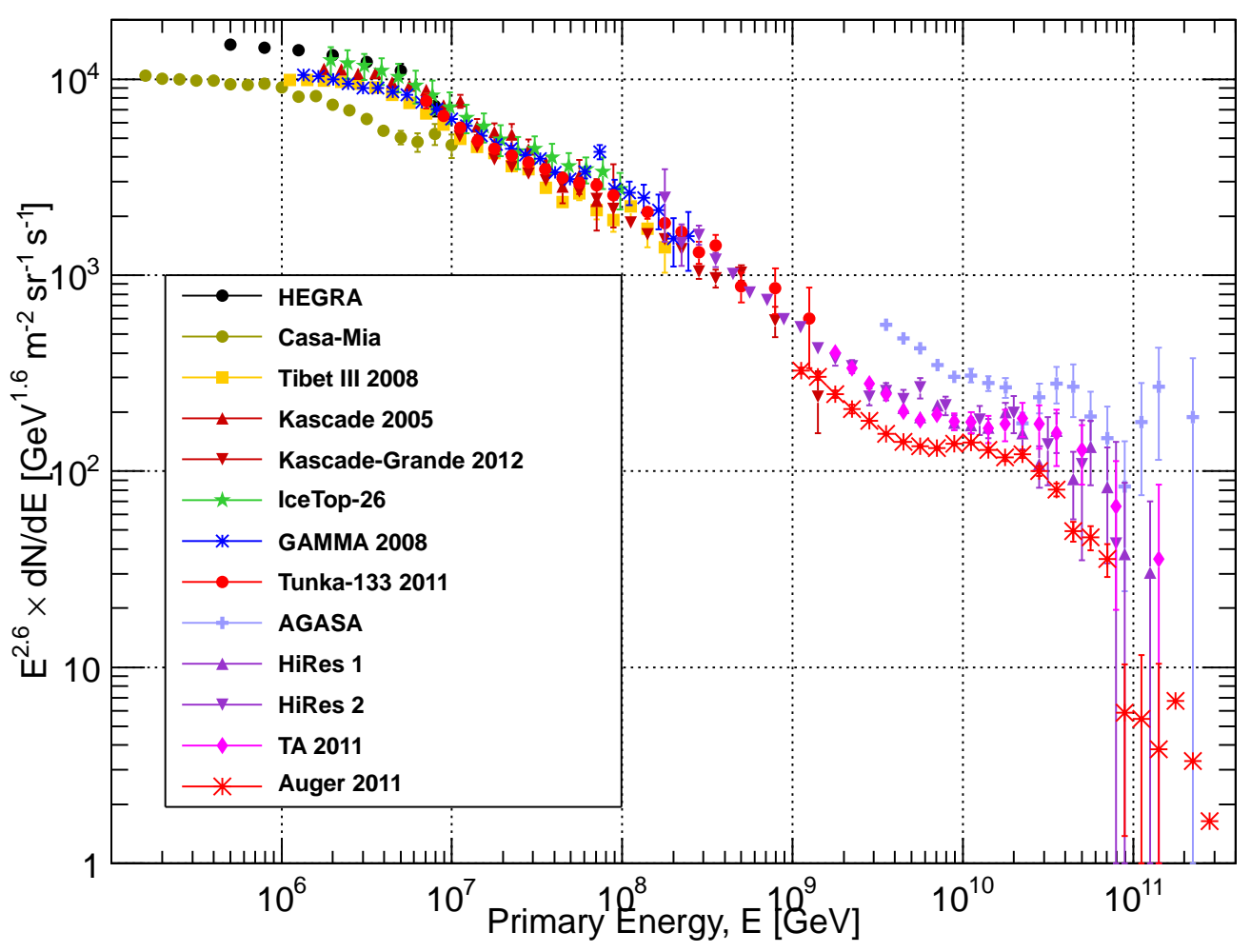

FIG. 1: All particle cosmic ray spectrum from air shower experiments. (References in text.)

defined as

$$
R=\frac{P c}{Z e},
$$

where $P$ is the total momentum of a nucleus and $Z e$ its electrical charge. Particles with the same rigidity and injection vector follow identical trajectories in a given magnetic field configuration. Rigidity is therefore the appropriate variable for interpreting changes in spectrum due to propagation and acceleration in magnetic fields. In particular, as first pointed out by Peters [14], if there is a maximum energy to which protons can be accelerated in a source, then the protons will cutoff first, followed by helium, carbon, .... according to

$$
E_{\max }(Z)=Z e \times R_{c}=Z \times E_{\max }(Z=1) .
$$

The outline of the paper is as follows. We first describe briefly the different types of air shower experiments and summarize the data from each of the selected measurements. We then use features in the energy spectrum observed by different experiments to construct a tentative all-particle energy spectrum from $10^{14}$ to $10^{20} \mathrm{eV}$. The most prominent features are the knee around $3 \times 10^{15} \mathrm{eV}$ and the ankle around $10^{19} \mathrm{eV}$, both prominent in Fig. 1 As justification for this approach, we note that, to a high degree of accuracy, there must be a single spectrum at
Earth. In the third major section of the paper we use this constructed all-particle spectrum as a template for discussing measurements of composition and possible implications for different sources. In this section we will assume the validity of the Peters cycle as written in Eq. 2 as a constraint on the energy dependence of different nuclear components. We describe two fits to the data, each of which has three populations of particles with contrasting assumptions about the rigidity cutoff for each population. The first two populations represent cosmic rays from galactic sources and the third population is an extragalactic component. Each population contains several groups of nuclei with assumed spectral indices as adjustable parameters.

\section{AIR SHOWER EXPERIMENTS}

Air shower detectors fall into several categories depending on the type of sensors used and on the altitude of the array. Scintillators such as those used in the Akeno array detect charge particles, which are mostly electrons and positrons with a fraction of order $10 \%$ of muons. In some arrays, the muon component can be distinguished from the electromagnetic component, either by a second layer of scintillator with an absorber between the top and 


\begin{tabular}{|l|r|r|r|}
\hline Array & Overburden $\left(\mathrm{g} / \mathrm{cm}^{2}\right.$ & type & Energy range \\
\hline \hline Tibet [22] & 559 & Scintillator & $1-200 \mathrm{PeV}$ \\
\hline Akeno [15] & 909 & Scintillator/muon & $0.5 \mathrm{PeV}-5 \mathrm{EeV}$ \\
\hline AGASA [31] & 909 & Scintillator & $4-200 \mathrm{EeV}$ \\
\hline HEGRA [21] & 755 & Air Cherenkov & $0.5-10 \mathrm{PeV}$ \\
\hline TUNKA [23] & 938 & Air Cherenkov & $7-1000 \mathrm{PeV}$ \\
\hline Kascade [17] & 1022 & Scintillator/muon & $2-90 \mathrm{PeV}$ \\
\hline Kascade-Grande [38] & 1022 & Scintillator/muon & $1-1400 \mathrm{PeV}$ \\
\hline GAMMA [37] & 700 & Scintillator/muon & $3-200 \mathrm{PeV}$ \\
\hline IceTop [19] & 680 & Ice Cherenkov & $1-1000 \mathrm{PeV}$ \\
\hline IceCube [54] & 680 & Ice Cherenkov (surface) + deep muons & $1-1000 \mathrm{PeV}$ \\
\hline CASA-MIA [16] & 860 & Scintillator + muon counters & $0.1-10 \mathrm{PeV}$ \\
\hline Fly's Eye [24] & 860 & Air Fluorescence & $1-100 \mathrm{EeV}$ \\
\hline Hi-Res [29] & 845 & Air Fluorescence & $0.2-100 \mathrm{EeV}$ \\
\hline Telescope Array [34] & 845 & Hybrid & $2-140 \mathrm{EeV}$ \\
\hline Auger [18] & 845 & Hybrid & $1-280 \mathrm{EeV}$ \\
\hline
\end{tabular}

TABLE I: List of selected air shower detectors. Hybrid refers to air fluorescence telescopes looking over a surface array.

bottom layers (as at Akeno [15]) or by separate, shielded, muon detectors (as in CASA-MIA [16] and Kascade [17]). The surface array at Auger [18] and IceTop [19] both use tanks filled with water or ice to detect the Cherenkov radiation produced by charged particles in the tanks. The method, pioneered in the Haverah Park array [20], is sensitive to photons in the shower that convert in the tanks as well as to the less numerous $e^{ \pm}$and muons.

HEGRA [21] and TUNKA [23] both use unshielded photomultipliers looking up at the night sky to detect the atmospheric Cherenkov light generated by the atmospheric cascade. In these arrays the depth of maximum, and hence the chemical composition of the cosmic rays, is judged by the lateral distribution of the Cherenkov light at the ground. The atmospheric fluorescence technique, first successfully used by Fly's Eye [24], traces the isotropically emitted nitrogen fluorescence lines excited by the passage of charged particles through the atmosphere. This method, after correcting for propagation through the atmosphere, maps the longitudinal development of each shower in the atmosphere. It therefore comes closest to providing a direct, calorimetric measurement of the energy of each event. By tracing each shower profile, a depth of maximum is assigned to each event. The distribution of depths of maximum is sensitive to primary composition. The atmospheric Cherenkov detectors provide depth of maximum and composition information in the region of the knee up to about $100 \mathrm{PeV}$, while the Fly's Eye type detectors provide this information around $1 \mathrm{EeV}$ and above.

A partial list of air shower detectors and information about them is given in Table \ An account of the history of the air shower method and the development of the various techniques is given in the review of Kampert \& Watson [25]. The current status of spectrum and composition is reviewed in the paper of Kampert \& Unger [26].

\section{FINDING A SINGLE ALL-PARTICLE SPECTRUM}

The technique of using a feature of the energy spectrum to inter-calibrate different measurements was emphasized by Berezinsky, Gazizov \& Grigorieva in their model of the ankle 27]. They explain the ankle (which appears as a "dip" when the spectrum is plotted as $\mathrm{E}^{3} \mathrm{dN} / \mathrm{dE}$ ) as being the result of energy loss by protons from sources at cosmological distances to $e^{ \pm}$pair production in the cosmic microwave background (CMB). They compared measurements of Yakutsk [28], a preliminary version of HiRes data [29], and a combination of Akeno (high energy) [30] and AGASA [31], shifting the energy scales respectively by $0.73,1.2$ and 0.9 . In a recent review [32], this approach is refined, extended to include more recent data 33, 34], and compared to three different models of the transition from Galactic to extragalactic cosmic rays. At lower energy, the position of the knee has been used to provide relative calibrations of different measurements in the energy range from $3 \times 10^{14}$ to $\sim 10^{16} \mathrm{eV}[35]$.

In this section, we show air shower data in two overlapping energy regions, $10^{14}$ to $10^{18} \mathrm{eV}$ and $10^{16}$ to $10^{21} \mathrm{eV}$. The first includes the knee and the second the ankle and the end of the spectrum. In these plots the lines show the all particle spectrum proposed in Ref. [36], which we discuss in the following section. It is important to keep in mind the amplification that occurs when data are plotted as $E^{\gamma+1} \mathrm{~d} N / \mathrm{d} E$, as is commonly done to display relatively small deviations from the underlying power law structure of the cosmic-ray spectrum. Air shower experiments typically measure and present there results as number of events per logarithmic bin of energy. Uncertainties in reconstructed energy generally scale with energy, so the resolution is expressed as $\delta E / E=\delta \ln (E)$. If the energy scale is shifted by an amount $\delta \ln (E)$, then each point moves on a log-log plot by a distance $\sqrt{1+\gamma^{2}} \delta \ln (E)$ at an angle elevated from 

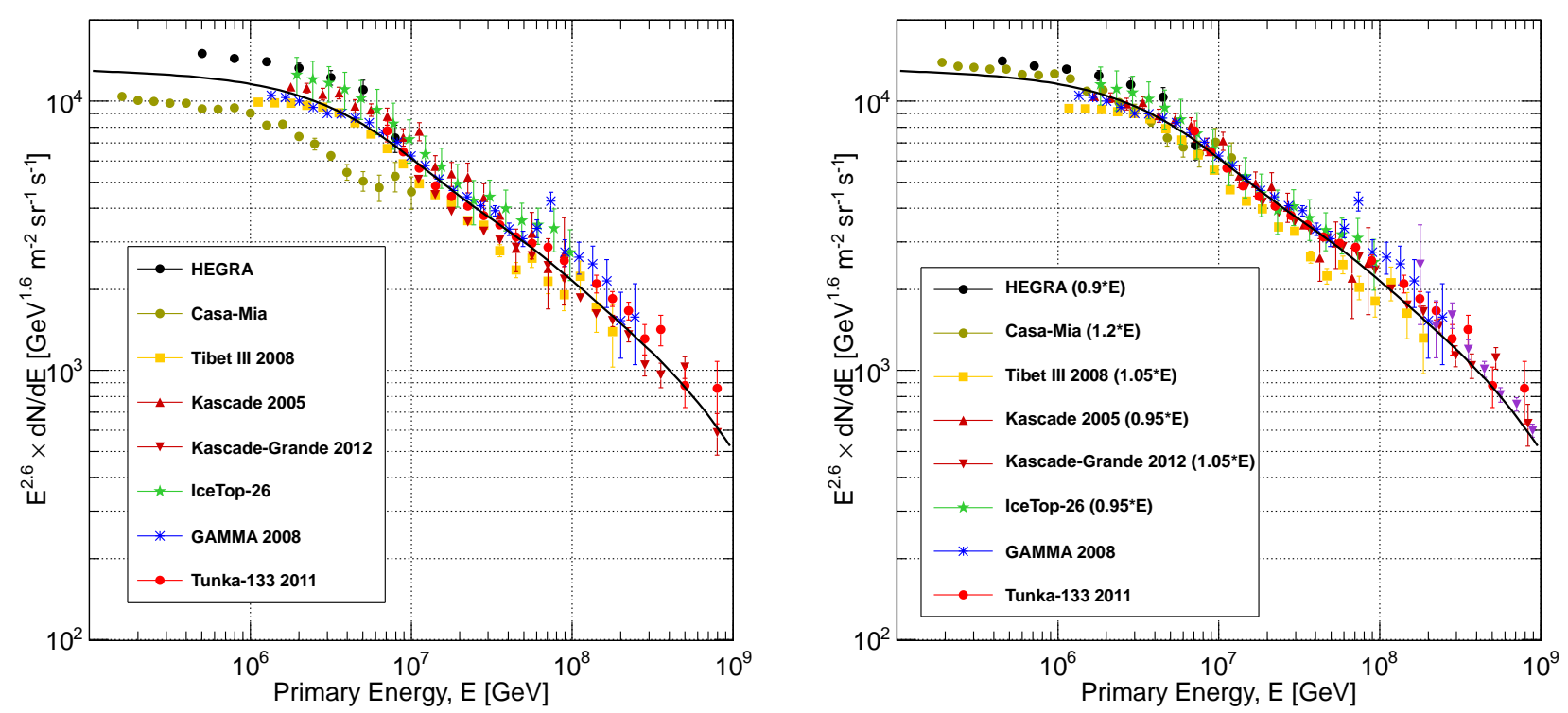

FIG. 2: Data from kilometer-scale air shower experiments for the spectrum up to one EeV, including the knee of the spectrum. Left: data as presented; Right: data replotted with energies shifted as shown in the labels.

the horizontal by $\theta=\tan ^{-1}(\gamma)$. For $\gamma=1.6$ as in Fig. 2 the shift is amplified by 1.89 and for $\gamma=2$ as in Fig. 3 the factor is 2.24. Thus, differences among data sets are not as large as they appear when multiplied by a power of the energy.

\section{A. The knee region}

To study the knee region we select several measurements that have similar (though not identical) structure to each other, but are offset to some extent. The left panel of Fig. 22 shows measurements of CASA-MIA [16], KASCADE [17], HEGRA [21] and Tibet 22], all of which show a bending corresponding to the knee of the spectrum. Three higher energy measurements, which start above the knee and extend to $10^{9} \mathrm{GeV}$ (Tunka [23], GAMMA [37] and KASCADE-Grande [38]) are also included. Tibet, being a closely spaced array at high altitude has data down to $100 \mathrm{TeV}$. CASA-MIA was also a closely spaced array at intermediate altitude.

The right panel of Fig. 2 shows the spectra replotted with energies shifted by factors as noted in the labels on the plot. Some differences in shape remain (particularly between CASA-MIA and Tibet in the knee region), but it is possible with moderate shifts to bring the data into substantially better agreement. Since the location of the knee is not fixed a priori, it is not possible to decide which experiment has the best absolute calibration.

\section{B. Ankle region}

Data up to the highest energies are collected in Fig. 3 . Data in the region of the ankle are from the giant air shower detectors AGASA [31], HiRes 1 and 2 [29], Auger [33] and Telescope Array [34]. The data below $10^{9} \mathrm{GeV}$ from Tunka, GAMMA and KASCADE-Grande are also included. The line shown is the model of Reference [36] with an extragalactic proton component. Larger shifts are needed to bring the different measurements into better agreement than in the case of the knee region. In their recent review [32], the Auger data has been shifted up by a factor 1.22 , which we also use. This brings the data into agreement in the decade between $10^{9}$ and $10^{10} \mathrm{GeV}$.

\section{APPROACHES TO DESCRIBING THE SPECTRUM}

Although it is possible to make a model in which the entire observed cosmic-ray spectrum comes from sources in our galaxy [39], it is generally accepted that the knee is in some way associated with the beginning of the end of a population particles accelerated by sources in the Milky Way and that the highest energy particles are from extragalactic sources. In this section we explore two rather different realizations of this basic idea. In both cases we follow the reasoning of Peters [14] by assuming that the knee and other features of the primary spectrum depend on magnetic rigidity as defined in Eq. 1. The motivation for this assumption is that both acceleration and 

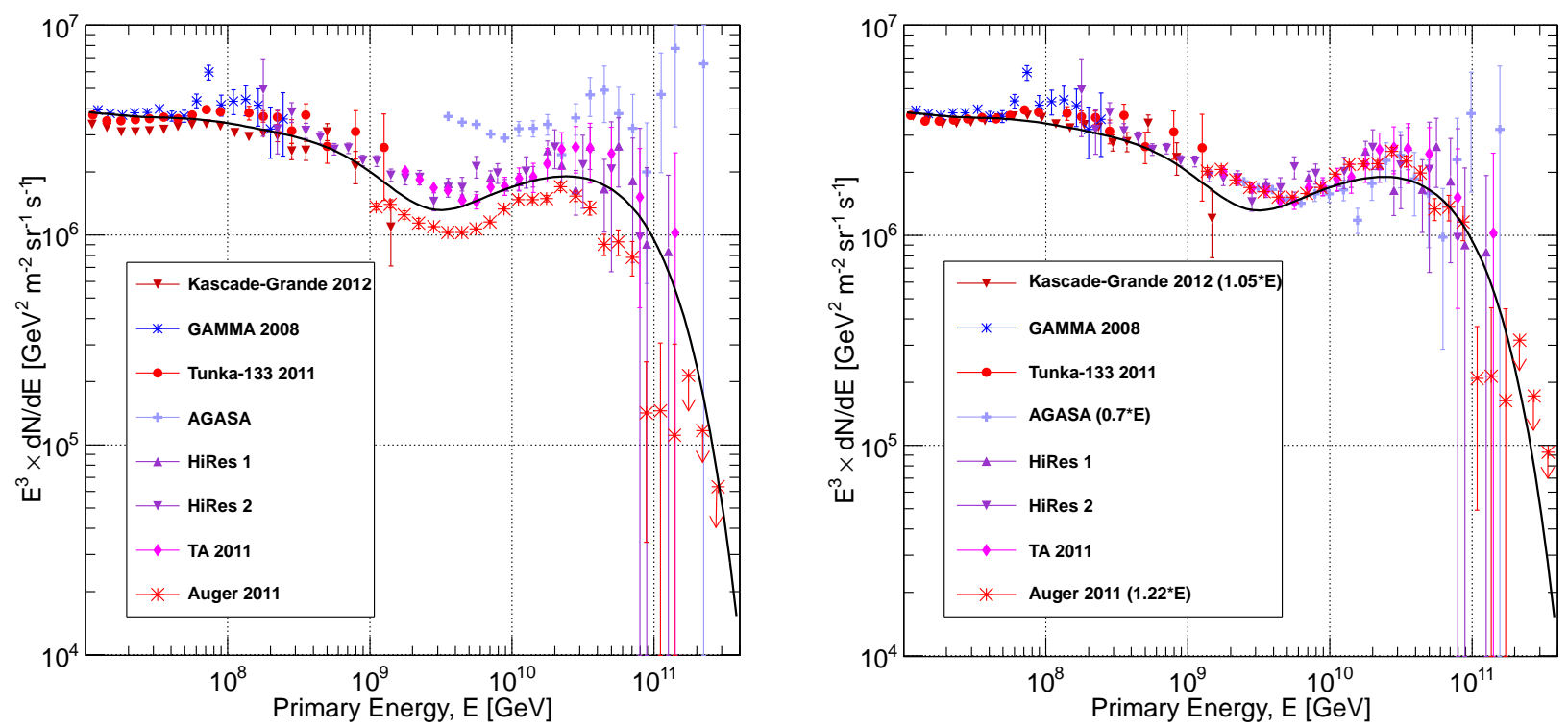

FIG. 3: Data from giant air shower detectors. Left: Data from AGASA [31], HiRes [29], Auger [18] and Telescope Array [34]; Right: same with data of AGASA shifted down in energy by 0.7 and Auger shifted up in energy by 1.22 .

propagation in models that involve collisionless diffusion in magnetized plasmas depend only on rigidity. The first evidence for a Peters cycle associated with the knee of the cosmic-ray spectrum comes from the unfolding analysis of measurements of the ratio of low-energy muons to electrons at the sea level with the KASCADE detector [17]. They found that the knee occurred earlier for protons and helium and later for heavier nuclei. The same Peters cycle pattern seems to occur also in the hardening of spectrum observed recently around $200 \mathrm{GV}$ as reported in Refs. [8] and [9].

\section{A. Hillas model}

The model of Ref. [36] is an attempt to implement the model of Hillas [40] in which the knee represents the end of the spectrum of cosmic rays accelerated by supernova remnants in the Milky Way and the ankle represents the transition to particles from extra-galactic sources. This picture depends on the amplification of magnetic fields by the turbulence associated with non-linear diffusive shock acceleration [41]. Support for the presence of magnetic field amplification by a factor of 100 above the level the interstellar medium comes from the narrow rims of synchrotron radiation by electrons observed at the edges of some SNR [42]. With fields of order $100 \mu$ Gauss, acceleration of protons to energies $E_{\max } \sim 3 \times 10^{6} \mathrm{GeV}$ is possible given the size and expansion rate of SNR [43]. In this situation it is natural to associate the knee with the maximum energy for the bulk of the galactic cosmic rays.

If the ankle signals the transition to extragalactic cos-

\begin{tabular}{|c|c|c|c|c|c|}
\hline & $\mathrm{p}$ & $\mathrm{He}$ & $\overline{\mathrm{CNO}}$ & $\mathrm{Mg}-\mathrm{Si}$ & $\mathrm{Fe}$ \\
\hline Pop. 1: & 7860 & 3550 & 2200 & 1430 & 2120 \\
\hline$R_{c}=4 \mathrm{PV}$ & 1.661 & 1.58 & 1.63 & 1.67 & 1.63 \\
\hline Pop. 2: & 20 & 20 & 13.4 & 13.4 & 13.4 \\
\hline$R_{c}=30 \mathrm{PV}$ & 1.4 & 1.4 & 1.4 & 1.4 & 1.4 \\
\hline Pop. 3: & 1.7 & 1.7 & 1.14 & 1.14 & 1.14 \\
\hline$R_{c}=2 \mathrm{EV}$ & 1.4 & 1.4 & 1.4 & 1.4 & 1.4 \\
\hline Pop. 3(*): & 200 & 0.0 & 0.0 & 0.0 & 0.0 \\
\hline$R_{c}=60 \mathrm{EV}$ & 1.6 & & & & \\
\hline
\end{tabular}

TABLE II: Cutoffs, normalization constants $\left(a_{i, j}\right)$ and integral spectral indexes $\left(\gamma_{i, j}\right)$ for Eq. 3 for the implementation of the Hillas model (H3a) in which all populations are mixed. In the bottom part of the table population $3\left({ }^{*}\right)$ consists of protons only (H4a).

mic rays, and the cutoff for the SNR component occurs at a rigidity of several $\mathrm{PV}$, then there is a gap between the knee and the ankle that has to be filled in by a higher energy galactic component, which Hillas calls "component B." In this case there would be at least three populations of particles. There could of course be many more components in a more realistic picture in which different classes of sources, or even individual sources have different individual characteristics. For this reason a three population model is a minimal assumption in case the transition to extra-galactic cosmic rays occurs at the ankle.

This three population picture is implemented in the model of Ref. [36] by assuming that each of the three components $(j)$ contains all five groups of nuclei and cuts off exponentially at a characteristic rigidity $R_{c, j}$. Thus 

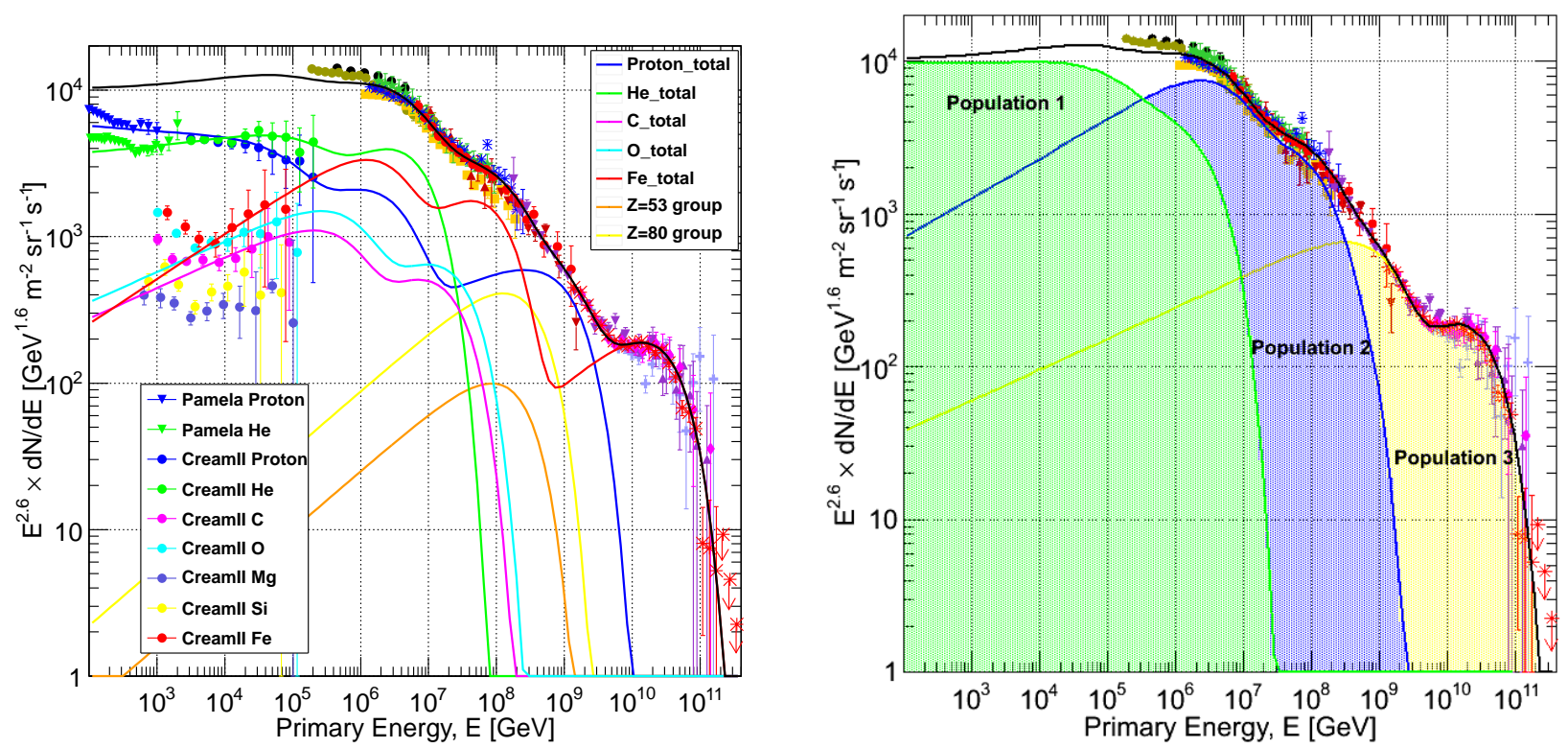

FIG. 4: Overview of the spectrum from below the knee to the ankle with the fit of Table III Air shower data shifted as in Figs. 2 and 3. Left: lines showing individual groups of nuclei from all populations compared to data from PAMELA [9] and CREAM [7] at low energy. Right: shaded regions show the overlapping contributions of the three populations.

the all-particle spectrum is given by

$$
\phi_{i}(E)=\Sigma_{j=1}^{3} a_{i, j} E^{-\gamma_{i, j}} \times \exp \left[-\frac{E}{Z_{i} R_{c, j}}\right] .
$$

The spectral indices for each group and the normalizations are given explicitly in Table II The parameters for Population 1 are from Refs. [7, 8], which we assume can be extrapolated to a rigidity of $4 \mathrm{PV}$ to describe the knee. In Eq. $3 \phi_{i}$ is d $N / \operatorname{dln} E$ and $\gamma_{i}$ is the integral spectral index. The subscript $i=1,5$ runs over the standard five groups (p, He, CNO, Mg-Si and Fe), and the all-particle spectrum is the sum of the five. This model is plotted as the solid line in Figs. 2 and 3 ,

\section{B. An alternative picture and global fit}

Spectra for the second fit are given by the same Eq. 3 but with qualitatively different parameters, as given in Table III. In particular, the first population has a much lower cutoff of $R_{c}=120 \mathrm{TV}$. This description is related to the significantly harder spectra assumed for the first population. Each component in the first population is fitted only above $R_{c}=200 \mathrm{GV}$, after the spectra hardening noted in Refs. 8] and [9]. With these harder spectra (as compared to Table 【i), the heavy components cannot be extended past the knee region. It is interesting to note that $R_{c} \approx 100 \mathrm{TV}$ is the classical result for the expected maximum energy of supernova remnants expanding into the interstellar medium with an un-amplified magnetic field of a few $\mu$ Gauss [44].
The spectrum with the parameters of Table III is shown in Fig. 4 from below the knee to the ankle. The contributions of individual groups of nuclei are shown, as well as the spectra of nuclei from CREAM [8]. We note that the bump in the spectrum around $10^{17} \mathrm{eV}$ corresponds with the "iron knee" reported by KASCADEGrande in their electron rich sample [45] and also noted by GAMMA [37]. A tendency for increasing mass above the knee has been noted for a long time (for example by CASA-MIA [46]), which seems now to be confirmed with higher resolution.

Another noteworthy feature is the possibility illustrated in this fit of explaining the ankle as a Peters cycle containing only protons and iron. This possibility is also suggested in Ref. [32] as an example of their "disappointing" model [47] of the end of the cosmic-ray spectrum. Such a picture is disappointing because the end of the spectrum would correspond to the highest energy to which cosmic-ray acceleration is possible, rather than to the Greisen-Zatsepin-Kuz'min effect in which higher energy particles lose energy in interactions with the cosmic microwave background [48, 49].

\section{Comments on fitting with several populations}

In both fits above we refer to three populations of particles, with spectral indices for each nuclear component and a single characteristic maximum rigidity for each population. The latter assumption has the effect of making the composition become heavier as each population 


\begin{tabular}{|c|c|c|c|c|c|c|c|}
\hline & $\mathrm{p}$ & $\mathrm{He}$ & $\mathrm{C}$ & $\mathrm{O}$ & $\mathrm{Fe}$ & $50<Z$ & $<Z<$ \\
\hline Pop. 1: & 7000 & 3200 & 100 & 130 & 60 & & \\
\hline$R_{c}=120 \mathrm{TV}$ & 1.661 & 1.58 & 1.4 & 1.4 & 1.3 & & \\
\hline Pop. 2: & 150 & 65 & 6 & 7 & 2.3 & 0.1 & 0.4 \\
\hline$R_{c}=4 \mathrm{PV}$ & 1.4 & 1.3 & 1.3 & 1.3 & 1.2 & 1.2 & 1.2 \\
\hline Pop. 3: & 14 & & & & 0.025 & & \\
\hline$R_{c}=1.3 \mathrm{EV}$ & 1.4 & & & & 1.2 & & \\
\hline$\overline{\overline{P o p} .2^{*}:}$ & $\overline{150}$ & $\overline{65}$ & $\overline{66}$ & $\overline{7}$ & 2.1 & $\overline{0.1}$ & $\overline{0.53}$ \\
\hline$R_{c}=4 \mathrm{PV}$ & 1.4 & 1.3 & 1.3 & 1.3 & 1.2 & 1.2 & 1.2 \\
\hline Pop. 3*: & 12 & & & & 0.011 & & \\
\hline$R_{c}=1.5 \mathrm{EV}$ & 1.4 & & & & 1.2 & & \\
\hline Pop. $4^{*}:$ & 1.2 & & & & & & \\
\hline$R_{c}=40 \mathrm{EV}$ & 1.4 & & & & & & \\
\hline
\end{tabular}

TABLE III: Global Fit results for the cutoffs, normalization constants $\left(a_{i, j}\right)$ and integral spectral indexes $\left(\gamma_{i, j}\right)$ for Eq. 3 In the bottom part of the table $\left(^{*}\right)$ populations 2 and 3 are slightly modified to accommodate a Population 4 of protons to bring $<\ln (A)>$ down to the observed level in Fig. 5

approaches its maximum, as illustrated in the left panel of Fig. 5. Another important point is that the higher energy populations can contribute significantly to the flux in the region dominated by the lower population. The right panel of Fig. 4 shows the overlap of the three populations of the global fit of Table III

The hardening of the spectrum observed by PAMELA and CREAM around $200 \mathrm{GV}$ is suggestive of the onset of a new population [50]. In this interpretation, the Population 1 of our global fit would be a higher energy population which becomes dominant above $200 \mathrm{GV}$, but which still contributes significantly at lower energies. Other explanations have been suggested. For example, Ref. [51] suggests that the hardening reflects the concave spectrum characteristic of non-linear diffusive shock acceleration. In Ref. 52 it is suggested that a dispersion in the injection spectra of different SNR is responsible for the hardening of the spectrum. Reference [53] shows how the hardening of the spectrum could be attributed to a change in the type of turbulence responsible for diffusion of the cosmic rays.

A general feature illustrated by the various parameterizations discussed here is that a Peters cycle of cutoffs of elemental components with rather hard spectra before the cutoff can produce regions of the all-particle spectrum that can be described approximately by steeper power laws. The differential spectral index between $100 \mathrm{GeV}$ and one $\mathrm{PeV}$ is close to 2.6 while the index above the second knee, between $2 \times 10^{18}$ and $5 \times 10^{19} \mathrm{eV}$ is approximately 3.35. The individual spectra in the global fit of Table III for example, have differential indices below their cutoffs ranging from 2.2 to 2.4 (except for hydrogen and helium below $200 \mathrm{GV}$ ).

In the case of the ankle structure, there is one model in which the absolute energy of the feature is fixed by the physical assumptions of the model. That is the original work of Berezinsky et al. [27], which explains the dip in the plot of $E^{3} \mathrm{~d} N / \mathrm{d} E$ as a consequence of physical process of pair production by protons during propa- gation through the cosmic microwave background radiation (CMB), which fixes the energy scale. In this "dip" model, the extragalactic spectrum extends below the ankle and the galactic-extragalactic transition occurs below one $\mathrm{EeV}$. In this case, according to Ref. [32] there is no need for a second, higher energy galactic component B. However, in order to avoid a gap in the energy spectrum around $10^{17} \mathrm{eV}$, the knee population would have to extend to significantly higher the $R_{c} \approx 4 \mathrm{GV}$ as in both fits in this paper.

The different populations of particles presumably correspond to different classes of sources. For this reason it is instructive to compare the energy content of the different populations of particles and estimate the power required at the source. As is well known, the total energy in the cosmic-ray spectrum of galactic cosmic rays, which is dominated by particles with energy below a $\mathrm{TeV}$, can be provided by supernova explosions at the rate of 3 per century. The assumption is that approximately $10 \%$ of the kinetic energy released goes into acceleration of cosmic rays, presumably by non-linear, first order, diffusive shock acceleration. With $10^{51} \mathrm{erg}$ in kinetic energy of the ejecta per supernova explosion, the total power into cosmic rays is then $\sim 3 \times 10^{50} \mathrm{erg} /$ century or $10^{41} \mathrm{erg} / \mathrm{s}$.

It is interesting to compare the power requirement for the second galactic population in the two models described above with the total power of the galactic cosmicray sources. To estimate this from the parameterizations of Tables II and III we start from a simplified version of the diffusion equation,

$$
N(E)=Q(E) \times \tau_{\text {esc }}(E) .
$$

Here $N(E)$ is the density of cosmic-ray particles (differential in energy) and $Q(E)$ is the number of particles per second per unit volume at which the sources inject particles of energy $E$. $\tau_{\text {esc }}(E)=\tau_{0} E^{-\delta}$ is the energy dependent escape time from the galaxy. We assume $\tau_{0}=10^{7}$ yrs and $\delta=0.33$. 62. Multiplying by the factor $4 \pi / c$, which converts flux to density, we can 

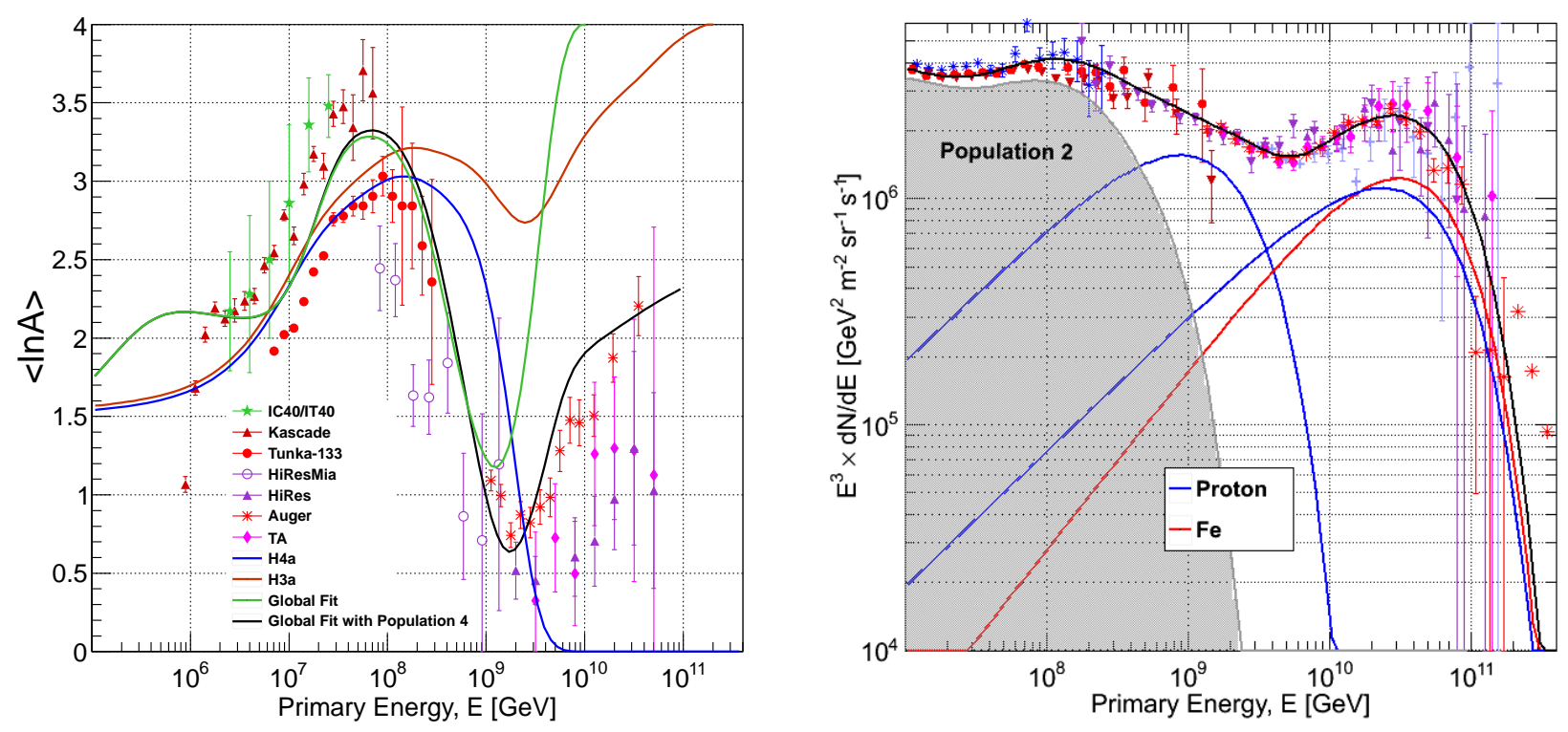

FIG. 5: Left: Mean $\ln (A)$ for the four parameterizations of tables $\Pi$ and $\Pi$, (For iron $\ln (A) \approx 4$.) Right: A modified fit with the addition of a 4 th population of extra-galactic protons (see text for discussion).

then integrate the spectra for Population 2 given the parameters in the Tables. We find $6 \times 10^{48} \mathrm{erg} /$ century for Component B (Population 2) of the Hillas model as parameterized in Table II and $2 \times 10^{49} \mathrm{erg} /$ century for Population 2 of the global fit of Table III] Both are reasonable in the sense that they could be accounted for by special sources at a level less than $10 \%$ of the total power requirement for all galactic cosmic rays.

\section{Composition}

Determination of composition with indirect air shower experiments is difficult, primarily because of the large fluctuations from shower to shower, which tend to smear out differences arising from the different mixture of primaries. Three data samples are shown in the left panel of Fig. [5 below $10^{18} \mathrm{eV}$. TUNKA results [23] are from measurement of the lateral distribution of Cherenkov light on the ground. IceCube results are from an analysis of one month of data on coincident events obtained when the detector was half complete [54]. Coincident events are those in which nearly vertical showers are reconstructed both by IceTop on the surface and by the deep detectors of IceCube more than $1.5 \mathrm{~km}$ below the surface. The composition-sensitive parameter is the ratio of the $\sim \mathrm{TeV}$ muons in the shower core to the shower size at the surface. Heavier primaries produce more light due to muon energy loss in the deep detector for a given shower size at the surface compared to light primaries. The KASCADE result [17] is based on measurements of the ratio of electrons to muons in showers at the surface, and we take the $<\ln (A)>$ values as plotted in Ref. [26].

In their review [26] Kampert \& Unger interpolated the depth of maximum measurements of several air fluorescence detectors between predictions for a pure proton assumption and a pure iron assumption in order to obtain a value for $<\ln (A)>$. We show in the left panel of Fig. [5] the values they inferred from HiRes-MIA [55], Auger and Telescope array. The results depend to some extent on the hadronic interaction model used to calculate depth of maximum for protons and iron, but the trend of the data is similar in different models. We plot their results for the SIBYLL model [56].

An important early result was obtained by the prototype of the HiRes fluorescence telescope observing showers in coincidence with the underground muon array that formed the muon detector for CASA-MIA. The combined hybrid detector allowed the profile of nearby, relatively small showers to be reconstructed. The measured depth of maximum distribution they observed [55] showed the depth of maximum increasing rapidly from $10^{17} \mathrm{eV}$ to $3 \times 10^{18} \mathrm{eV}$ in a manner consistent with all the parameterizations in the left panel of Fig. 5 except the disappointing model with only iron at the highest energies.

There is a significant disagreement in interpretation of depth of maximum measurements above $10^{18} \mathrm{eV}$, with Hi-Res and TA preferring nearly pure protons and Auger preferring a transition to heavies. But in both cases, a transition to pure iron, as would be the case at the end of the spectrum in the case of the disappointing model, does not seem to be indicated. The right panel of Fig. 5 shows a fit that contains a 4 th population made entirely of protons to show the amount that would be needed to bring the composition closer to the data in this energy 
region. There would need to comparable amounts of protons and iron. We note that such a mixture would not be consistent with the small fluctuations seen by Auger [57], which would seem to require a more pure composition of heavy nuclei. It appears that there is not at present a satisfactory understanding of the highest energy cosmic rays.

\section{CONCLUSION}

\section{A. Transition from galactic to extragalactic sources}

The energy range were Populations 2 and 3 meet is usually treated as the transition from galactic to extragalactic cosmic ray sources. There are at least three different ways in which this transition region can be treated.

In the dip model [27] the transition region is below $10^{18} \mathrm{eV}$. The dip itself is generated by the energy loss on production of electron-positron pairs in interactions on the microwave background. The energy spectrum of the extragalactic protons is flatter below $10^{18} \mathrm{eV}$. What that means is the transition from heavy galactic nuclei to extragalactic protons and He nuclei happens below that energy. This is very different from the classical 'ankle' model [58] where the transition region is above that energy and the galactic cosmic ray contribution above $10^{19}$ $\mathrm{eV}$ is significant. The chemical composition of cosmic rays then starts changing significantly only above $10^{19}$ $\mathrm{eV}$.

The third model is that of mixed cosmic ray composition at the highest energies [59]. In such models the extragalactic cosmic rays are accelerated with a chemical composition similar to that of the $\mathrm{GeV}$ galactic cosmic rays. Their composition is changed in propagation because of photoproduction, electron positron pair creation, and nuclear photodisintegration. The transition between galactic and extragalactic cosmic rays is at intermediate energy, and the final energy spectrum and chemical composition depend strongly on the distance to the extragalactic sources.

\section{B. Outlook}

As noted in the previous paragraphs, there is much interest in studying the transition from galactic to extragalactic cosmic rays, which is expected in the energy range between $10^{17}$ and $10^{19} \mathrm{eV}$. There is a lack of data at present because this energy range is at the upper end of the range of kilometer-scale experiments such as TUNKA, KASCADE-Grande and IceTop, and it overlaps the threshold region for the giant air shower detectors, Auger and Telescope Array. As a consequence, we do not yet have a good understanding of this important transition region. Only preliminary results from IceCube and IceTop have been presented so far. With the completion of the detector and operation with the full detector since May, 2011, we can expect to see significant new results from IceCube at the beginning of the transition region, but the kilometer-scale detectors will always be statistically limited above the EeV energy range. From the higher energy side, the big experiments are working to decrease their energy threshold and become efficient down to the $10^{17} \mathrm{eV}$ energy range.

The Auger Southern Observatory has added the High Elevation Auger Telescope (HEAT, [60]) and the Auger Muon and Infill for the Ground Array (AMIGA, 61]) to the original design in order to lower their threshold. Air showers of energy around $10^{17} \mathrm{eV}$ do not emit sufficient light to be seen further than a few kilometers away. At that distance the depth of maximum $X_{\max }$ appears at higher elevation, outside of the viewing angle of the original design. In addition, nearby showers of lower energy reach their maximum of development above the $30^{\circ}$ limit of the original Auger telescopes. HEAT is composed of three fluorescence telescopes of the same basic design as the original Auger telescope and is installed at the western fluorescence detector site of the observatory. They can operate in two positions. Horizontally they share the same field of view as the original telescopes. This position is used for calibration of the instruments. Tilted upward by $29^{\circ}$, which is the normal operation mode for HEAT, they observe the upper part of the atmosphere. The first light from one of those telescopes was seen in January 2009.

An infill array of 85 detectors is deployed on two grids of one half $(750 \mathrm{~m})$ and one fourth $(433 \mathrm{~m})$ of the regular Auger surface array grid over the field of view of HEAT. The $750 \mathrm{~m}$ infill covers an area of $23.5 \mathrm{~km}^{2}$ and the 433 $\mathrm{m}$ infill covers $5.9 \mathrm{~km}^{2}$. There are also plans to have muon counters in AMIGA. The close spacing of the new subarray is $10^{17} \mathrm{eV}$.

The Telescope Array experiment is in the process of building its low energy extension TALE (G. Thomson, private communication). It will consist of ten fluorescent telescopes located at the Northern side of the array. These telescopes will be elevated to be sensitive from $31^{\circ}$ to $59^{\circ}$ above the horizon. One hundred new scintillator counters will be deployed nearby at distances of $400 \mathrm{~m}$ from each other $(1 / 3$ of the standard spacing in the Telescope Array). Thirty-five of them will be operational in March 2013. TALE will be sensitive to primary particles of energy above $10^{16.5} \mathrm{eV}$.

In summary, a thorough study with good statistics of the energy region between $10^{17}$ and $10^{19} \mathrm{eV}$ should be scientifically productive.
[1] "Cosmic ray lifetime in the Galaxy - experimental results and models," J.A. Simpson, \& M. Garcia-Muñoz, Space
Science Reviews 46, 205-224 (1988). 
[2] "Nonlinear theory of diffusive acceleration of particles by shock waves," M. A. Malkov \& L. O'C Drury, Rep. Progr. Phys., 64, 421-489 (2001). Reference for diffusive shock acceleration by SNR.

[3] "The boron-to-carbon abundance ratio and Galactic propagation of cosmic radiation," A. Obermeier, P. Boyle, J. Hörandel \& D. Müller Ap.J., 752, 69 (2012).

[4] "Fermi-LAT observations of the diffuse $\gamma$-ray emission: implications for cosmic rays and the interstellar medium," M. Ackermann et al., (Fermi Collaboration) Ap.J. 750, 3 (2012).

[5] "TeV gamma-ray astronomy; A summary," J. Holder Astropart.Phys. 39-40, 61 (2012).

[6] "Direct measurements of cosmic rays using balloon borne experiments," E.-S. Seo, Astropart. Phys. 39-40, 76 (2012)

[7] "Energy spectra of cosmic-ray nuclei at high energies," H.S. Ahn et al.(CREAM Collaboration), Ap.J. 707, 593603 (2009)

[8] "Discrepant hardening observed in cosmic-ray elemental spectra," H.S. Ahn et al., (CREAM Collaboration) Ap.J. 714, L89-L93 (2010)

[9] "PAMELA Measurements of Cosmic-Ray Proton and Helium Spectra," O. Adriani et al.(Pamela Collaboration), Science 332, 69 (2011).

[10] "Cosmic-ray Proton and Helium Spectra: Results from the JACEE Experiment," K. Asakimori et al., Ap.J. 502, 278 (1998)

[11] "Cosmic Ray Composition and Spectra: (II) Helium and Z > 2," K. Asakimori et al., Proc. 23rd Int. Cosmic Ray Conf., Calgary, 2, 25-29 (1993) and Proc. 22nd Int. Cosmic Ray Conf., Dublin, 2, 57 and 97 (1991).

[12] "Energy Spectra of Abundant Nuclei of Primary Cosmic Rays from the Data of ATIC-2 Experiment: Final Results," A.D. Panov et al. (ATIC Collaboration), Bull.Russ.Acad.Sci.Phys. 73, 564 (2009); arXiv:1101.3246

[13] "Studies of the energy spectra of high and ultra-high primary cosmic ray particles on the 'Proton' space stations," N.L. Grigorov et al., Yad. Fiz. 11, 1058 (1970) and Proc. 12th Int. Cosmic Ray Conf., Hobart, 2, 206 (1971).

[14] "Primary Cosmic Radiation and Extensive Air Showers," B. Peters, Il Nuovo Cim. XXII, 800-819 (1961).

[15] "Energy spectrum of primary cosmic rays between $10^{14.5}$ and $10^{18} \mathrm{eV}$," M. Nagano et al., J. Phys. G 10, 1295-1310 (1984).

[16] "The cosmic ray energy spectrum between $10^{14}$ and $10^{16}$ eV," M.A.K. Glasmacher et al., Astropart. Phys. 10, 291-302 (1999).

[17] "KASCADE measurements of energy spectra for elemental groups of cosmic rays: Results and open problems," T. Antoni et al. (Kascade Collaboration), Astropart. Phys. 24, 1 (2005).

[18] "Observation of the Suppression of the Flux of Cosmic Rays above $4 \times 10^{19} \mathrm{eV}$," J. Abraham et al. (Auger Collaboration), Phys. Rev. Lett. 101, 061101 (2008).

[19] "IceTop: the surface component of IceCube," R. Abbasi et al., (IceCube Collaboration) N.I.M. A 700, 188-220 (2013).

[20] "The cosmic ray energy spectrum above $4 \times 10^{17} \mathrm{eV}$ as measured by the Haverah Park array," M.A. Lawrence, R.J.O. Reid, \& A.A. Watson, J. Phys. G 17, 733-758 (1991).

[21] "Energy Spectrum and Chemical Composition of Cos- mic Rays between 0.3 and $10 \mathrm{PeV}$ determined from the Cherenkov-Light and Charged-Particle distributions in Air Showers," F. Arqueros et al., Astron. \& Astrophys. 359, 682 (2000).

[22] "The All-Particle Spectrum of Primary Cosmic Rays in the Wide Energy Range from $10^{14}$ to $10^{17} \mathrm{eV}$ Observed with the Tibet-III Air-Shower Array," M. Amenomori et al. (Tibet), Ap. J. 678, 1165-1179 (2008).

[23] "The Tunka-133 EAS Cherenkov light array: status of 2011," S.F. Berezhnev et al.(Tunka Collaboration), NIM A692, 98 (2012).

[24] "The cosmic-ray energy spectrum observed by the Fly's Eye," D.J. Bird et al., Astrophys. J. 424, 491-502 (1994).

[25] "Extensive air showers and ultra high-energy cosmic rays: a historical review," K.-H. Kampert \& A.A. Watson, Eur. Phys. J. H 37, 359-412 (2012).

[26] "Measurements of the Cosmic Ray Composition with Air Shower Experiments," K.-H. Kampert \& M. Unger, Astropart. Phys. 35, 660-678 (2012).

[27] "On astrophysical solution to ultrahigh energy cosmic rays," V. Berezinsky, A. Gazizov \& S. Grigorieva, Phys. Rev. D 74043005 (2006).

[28] "Muons in extensive air showers of energies $E_{0}=$ $10^{16.6} 10^{19.8} \mathrm{eV}, "$ A.V. Glushkov et al., (Yakutsk Collaboration), JETP Lett., 71, 97 (2000).

[29] "First Observation of the Greisen-Zastsepin-Kuzmin Suppression," R.U. Abbasi et al. (HiRes Collaboration), Phys. Rev. Lett. 100 (2008) 101101. See also "Measurement of the flux of ultra high energy cosmic rays by the stereo technique," R.U. Abbasi et al., Astropart. Phys. 32, 53-60 (2009).

[30] "Energy spectrum of primary cosmic rays above $10^{17.9} \mathrm{eV}$ determined from extensive air shower experiments at Akeno," M. Nagano et al., J. Phys. G 18, 423-442 (1992).

[31] "Energy determination in the Akeno Giant Air Shower Array experiment," M. Takeda et al. (The AGASA Collaboration), Astropart. Phys. 19, 447-462 (2003).

[32] "Transition from galactic to extragalactic cosmic rays," R. Aloisio, V. Berezinsky \& A. Gazizov, Astropart. Phys., 39-40, 129 (2012).

[33] "The Pierre Auger Observatory I: The Cosmic Ray Energy Spectrum and Related Measurements," P. Abreu et al. (Auger Collaboration), in Proc. 32nd Int. Cosmic Ray Conf., Beijing, China arXiv:1107.4809 (see Table at www.Auger.org).

[34] "The Cosmic Ray Energy Spectrum Observed with the Surface Detector of the Telescope Array Experiment," T. Abu-Zayyad et al., (Telescope Array Collaboration), arXiv:1205.5067

[35] "Structure in the cosmic ray spectrum: an update," A.D. Erlykin and A.W. Wolfendale, J. Phys. G: Nucl. Part. Phys. 27, 1005 (2001).

[36] "Spectrum of cosmic-ray nucleons, kaon production, and the atmospheric muon charge ratio," T.K. Gaisser, Astroparticle Physics 35 801-806 (2012).

[37] "An all-particle primary energy spectrum in the 3200 PeV energy range," A.P. Garyaka et al., J. Phys. G: Nucl. Part. Phys., 35, 115201 (2008)

[38] "The spectrum of high-energy cosmic rays measured with KASCADE-Grande," W.D. Apel et al., (KASCADEGrande Collaboration), Astropart. Phys., 36, 183-194 (2012).

[39] "Role of Galactic Sources and Magnetic Fields in Forming the Observed Energy-Dependent Composition of 
Ultrahigh-Energy Cosmic Rays," A. Calvez, A. Kusenko \& S. Nagataki, Phys. Rev. Letters 105, 091101 (2010).

[40] "Can diffusive shock acceleration in supernova remnants account for high-energy galactic cosmic rays?" A.M. Hillas, J. Phys. G: Nucl.Part.Phys., 31, R95 (2005)

[41] "Turbulent amplification of magnetic field and diffusive shock acceleration of cosmic rays," A.R. Bell, MNRAS, 353, 550-558 (2004).

[42] "Extremely fast acceleration of cosmic rays in a supernova remnant," Y. Uchiama et al., Nature 449, 576 (2007)

[43] "Non-linear particle acceleration at non-relativistic shock waves in the presence of self-generated turbulence," E. Amato \& P. Blasi, MNRAS 371, 1251-1258 (2006).

[44] "The maximum energy of cosmic rays accelerated by supernova shocks," P.O. Lagage \& C.J. Cesarsky, Astron. \& Astrophys. 125, 249-257 (1983).

[45] "Kneelike Structure in the Spectrum of the Heavy Component of Cosmic Rays Observed with KASCADEGrande," W.D. Apel et al., Phys. Rev. Lett. 107, 171104 (2011).

[46] "The cosmic ray composition between $10^{14}$ and $10^{16} \mathrm{eV}$," M.A.K. Glasmacher et al., Astropart. Phys. 12, 1-17 (1999).

[47] "Ultra High Energy Cosmic Rays: The disappointing model," Astropart. Phys. 34, 620-626 (2011).

[48] "End to the cosmic-ray spectrum?" K. Greisen, Phys. Rev. Letters 16, 748-750 (1966).

[49] "Upper Limit of the Spectrum of Cosmic Rays," G.T. Zatsepin \& Kuz'min, J.E.T.P Letters 4, 78-80 (1966).

[50] "A new component of cosmic rays?," A.D. Erlykin \& A.W. Wolfendale, Astropart. Phys. 35, 449-456 (2012).

[51] "Spectra of Cosmic Ray Protons and Helium Produced in Supernova Remnants," V. Ptuskin, V. Zirakashvili \& E-S. Seo, arXiv:1212.0381

[52] "Cosmic ray spectral hardening due to dispersion in the source injection spectra," Q. Yuan, B. Zhang \& X.-J. Bi,
Phys. Rev. D 84, 043002 (2011).

[53] "Spectral Breaks as a Signature of Cosmic Ray Induced Turbulence in the Galaxy," P. Blasi, E. Amato \& P.D. Serpico, Phys. Rev. Letters 109, 061101 (2012).

[54] "Cosmic Ray Composition and Energy Spectrum from $130 \mathrm{PeV}$ Using the 40-String Configuration of IceTop and IceCube," R. Abbasi et al.(IceCube Collaboration), Astropart. Phys. 42, 15-32 (2013).

[55] "Measurement of the cosmic-ray energy spectrum and composition from $10^{17}$ to $10^{18.3} \mathrm{eV}$ using a hybrid technique," T. Abu-Zayyad et al. (HiRes-MIA), Astrophys. J. 557, 686-699 (2001). See also "Evidence for Changing of Cosmic Ray Composition between $10^{17}$ and $10^{18} \mathrm{eV}$," T. Abu-Zayyad et al., (HiRes Collaboration), Phys. Rev. Lett. 84, 4276-4279 (2003).

[56] "Cosmic ray interaction event generator SIBYLL 2.1," E.-J. Ahn, R. Engel, T.K. Gaisser, P. Lipari \& T. Stanev, Phys. Rev. D 80, 094003 (2009).

[57] "Measurement of the Depth of Maximum of Extensive Air Showers above $10^{18} \mathrm{eV}, "$ J. Abraham et al., Phys. Rev. Letters 104, 091101 (2010).

[58] "Cosmological origin of cosmic rays of energy above $10^{19}$ eV," E. Waxman, Ap.J., 452, L1 (1995).

[59] "Extragalactic propagation of ultrahigh energy cosmic rays" D. Allard, Astropart. Phys. 39-40, 33 (2012).

[60] "The HEAT Telescope of the Pierre Auger Observatory," T.H-J. Mathes for the Auger Collaboration, Proc. 32nd ICRC (Beijing), paper 0761 (2011).

[61] "The AMIGA detector of the Pierre Auger Observatory: an overview," F. Sanchez for the Auger Collaboration, Proc. 32nd ICRC (Beijing), paper 0742 (2011).

[62] The measured ratio of secondary/primary nuclei at low energy decreases like $E^{-0.6}[3]$. Such a strong energy dependence cannot continue to the knee region without producing unobserved anisotropy in the cosmic radiation at high energy. 